\title{
Entire Functions of Bounded L-Index: Its Zeros and Behavior of Partial Logarithmic Derivatives
}

\author{
Andriy Bandura ${ }^{1}$ and Oleh Skaskiv ${ }^{2}$ \\ ${ }^{1}$ Department of Advanced Mathematics, Ivano-Frankivsk National Technical University of Oil and Gas, 15 Karpatska St., \\ Ivano-Frankivsk 76019, Ukraine \\ ${ }^{2}$ Department of Function Theory and Theory of Probability, Ivan Franko National University of Lviv, 1 Universytetska St, \\ Lviv 79000, Ukraine \\ Correspondence should be addressed to Andriy Bandura; andriykopanytsia@gmail.com
}

Received 5 August 2017; Accepted 15 October 2017; Published 6 November 2017

Academic Editor: Stanislawa Kanas

Copyright (C) 2017 Andriy Bandura and Oleh Skaskiv. This is an open access article distributed under the Creative Commons Attribution License, which permits unrestricted use, distribution, and reproduction in any medium, provided the original work is properly cited.

\begin{abstract}
In this paper, we obtain new sufficient conditions of boundedness of $\mathbf{L}$-index in joint variables for entire function in $\mathbb{C}^{n}$ functions. They give an estimate of maximum modulus of an entire function by its minimum modulus on a skeleton in a polydisc and describe the behavior of all partial logarithmic derivatives and the distribution of zeros. In some sense, the obtained results are new for entire functions of bounded index and $l$-index in $\mathbb{C}$ too. They generalize known results of Fricke, Sheremeta, and Kuzyk.
\end{abstract}

\section{Introduction}

In this paper, we find multidimensional sufficient conditions of boundedness of $\mathbf{L}$-index in joint variables, which describe distribution of zeros and behavior of partial logarithmic derivatives. Recently, we published a paper [1] where some similar restrictions are established. Another approach was used by a slice function $F\left(z^{0}+t \mathbf{b}\right)$, where $z^{0} \in \mathbb{C}^{n}, t \in$ $\mathbb{C}, \mathbf{b}$ is a given direction in $\mathbb{C}^{n} \backslash\{0\}, F: \mathbb{C}^{n} \rightarrow \mathbb{C}$ is an entire function. It is a background for concept of function of bounded $L$-index in direction (see definition and properties in $[2,3])$. We proved that if an entire function in $\mathbb{C}^{n}$ function $F$ is of bounded $l_{j}$-index in every direction $\mathbf{1}_{j}=$ $(0, \ldots, 0, \underbrace{1}_{j \text { th place }}, 0, \ldots, 0)$, then $F$ is of bounded $\mathbf{L}$-index in joint variables for $\mathbf{L}=\left(l_{1}, \ldots, l_{n}\right), l_{j}: \mathbb{C}^{n} \rightarrow \mathbb{R}_{+}$(Theorem 6 , [1]). It helped us to find restrictions by directional logarithmic derivatives and distribution zeros in every direction $\mathbf{1}_{j}, j \in$ $\{1, \ldots, n\}$. We assumed that the logarithmic derivative in direction $\mathbf{1}_{j}$ is bounded by a function $l_{j}$ outside some exceptional set, which contains all zeros of entire function $F$ (see definition of $G_{r}^{\mathbf{b}}(F)$ below). Prof. Chyzhykov paid attention in conversation with authors that this exceptional set is too small because it does not contain neighborhoods of some zeros of the function in $\mathbb{C}^{n}$. Thus, it leads to the following question: is there sufficient conditions of boundedness of $\mathbf{L}$ index in joint variables with larger exceptional sets? We give a positive answer to this question (Theorem 10). Moreover, we obtain sufficient conditions of boundedness of $\mathbf{L}$-index in joint variables by estimating the maximum modulus of an entire function on the skeleton in polydisc by minimum modulus (Theorem 7). Theorems 9 and 10 present restrictions by a measure of zero set of an entire function $F$, under which $F$ has bounded L-index in joint variables. Nevertheless, we do not know whether the obtained conditions in Theorems 7-10 are necessary too in $\mathbb{C}^{n},(n \geq 2)$. Note that these propositions are new even for entire functions of bounded index in joint variables, i. e. $\mathbf{L}=(1, \ldots, 1)$ (see definition and properties in [4-8]).

It is known [9] that for every entire function $f$ with bounded multiplicities of zeros there exists a positive continuity on $[0 ;+\infty)$ function $l(r)(r=|z|)$ such that $f$ is of bounded $l$-index. This result can be easily generalized for entire functions in $\mathbb{C}^{n}$. Thus, the concept of bounded $\mathbf{L}$-index in joint variables allows the study of growth properties of any entire functions with bounded multiplicities of zero points.

It should be noted that the concepts of bounded $L$-index in a direction and bounded $\mathbf{L}$-index in joint variables have few 
advantages in the comparison with traditional approaches to study properties of entire solutions of differential equations. In particular, if an entire solution has bounded index [10], then it immediately yields its growth estimates, a uniform distribution of its zeros, a certain regular behavior of the solution, and so forth. A full bibliography about application in theory of ordinary and partial differential equations is in $[3,11,12]$.

The paper is devoted to two old problems in theory of entire and meromorphic functions. The first problem is the establishment of sharp estimates for the logarithmic derivatives of the functions in the unit disc outside some exceptional set. Chyzhykov et al. [13-16] considered various formulations of the problem. The obtained estimates were used to study properties of holomorphic solutions of differential equations. Instead, the authors assume that partial logarithmic derivative in every variable satisfies some inequalities (28) or (45).

Another interesting considered problem concerns zero sets of holomorphic function in $\mathbb{C}^{n}$. The different estimates of measure of zero set and its geometrical properties are investigated in [17-22]. We suppose that zero points of entire functions admit uniform distribution in some sense, that is, (29).

Below we use results from Ukrainian papers [23, 24], but they are also included in English monographs [3, 11].

\section{Main Definitions and Notations}

We need some standard notations. Let $\mathbb{R}_{+}=[0,+\infty)$. Denote $\mathbf{0}=(0, \ldots, 0) \in \mathbb{R}_{+}^{n}, \mathbf{1}=(1, \ldots, 1) \in \mathbb{R}_{+}^{n}, \mathbf{2}=(2, \ldots, 2) \in \mathbb{R}_{+}^{n}$, $\mathbf{1}_{j}=(0, \ldots, 0, \underbrace{1}_{j \text { th place }}, 0, \ldots, 0) \in \mathbb{R}_{+}^{n}$.

For $R=\left(r_{1}, \ldots, r_{n}\right) \in \mathbb{R}_{+}^{n}$ and $K=\left(k_{1}, \ldots, k_{n}\right) \in \mathbb{Z}_{+}^{n}$ denote $\|R\|=r_{1}+\cdots+r_{n}, K !=k_{1} ! \cdot \ldots \cdot k_{n} !$. For $=\left(a_{1}, \ldots, a_{n}\right) \in$ $\mathbb{C}^{n}, b=\left(b_{1}, \ldots, b_{n}\right) \in \mathbb{C}^{n}, z=\left(z_{1}, \ldots, z_{n}\right) \in \mathbb{C}^{n}$, we will use formal notations without violating the existence of these expressions:

$$
\begin{aligned}
|a| & =\left(\left|a_{1}\right|,\left|a_{2}\right|, \ldots,\left|a_{n}\right|\right), \\
A \pm B & =\left(a_{1} \pm b_{1}, \ldots, a_{n} \pm b_{n}\right), \\
A B & =\left(a_{1} b_{1}, \ldots, a_{n} b_{n}\right), \\
\frac{A}{B} & =\left(\frac{a_{1}}{b_{1}}, \ldots, \frac{a_{n}}{b_{n}}\right), \\
A^{B} & =a_{1}^{b_{1}} a_{2}^{b_{2}} \cdot \ldots \cdot a_{n}^{b_{n}}, \\
d z & =d z_{1} d z_{2} \cdots d z_{n} .
\end{aligned}
$$

If $a, b \in \mathbb{R}^{n}$ the notation $a<b$ means that $a_{j}<b_{j}(j=$ $1, \ldots, n)$; similarly, the relation $a \leq b$ is defined.

The polydisc $\left\{z \in \mathbb{C}^{n}:\left|z_{j}-z_{j}^{0}\right|<r_{j}, j=1, \ldots, n\right\}$ is denoted by $\mathbb{D}^{n}\left(z^{0}, R\right)$, its skeleton $\left\{z \in \mathbb{C}^{n}:\left|z_{j}-z_{j}^{0}\right|=r_{j}, \quad j=\right.$ $1, \ldots, n\}$ is denoted by $\mathbb{T}^{n}\left(z^{0}, R\right)$, and the closed polydisc $\{z \in$ $\left.\mathbb{C}^{n}:\left|z_{j}-z_{j}^{0}\right| \leq r_{j}, j=1, \ldots, n\right\}$ is denoted by $\mathbb{D}^{n}\left[z^{0}, R\right]$.
For $K=\left(k_{1}, \ldots, k_{n}\right) \in \mathbb{Z}_{+}^{n}$ and partial derivatives of entire function $F(z)=F\left(z_{1}, \ldots, z_{n}\right)$ we will use the notation

$$
F^{(K)}(z)=\frac{\partial^{\|K\|} F}{\partial z^{K}}=\frac{\partial^{k_{1}+\cdots+k_{n}} f}{\partial z_{1}^{k_{1}} \cdots \partial z_{n}^{k_{n}}} .
$$

Let $\mathbf{L}(z)=\left(l_{1}(z), \ldots, l_{n}(z)\right)$, where $l_{j}(z)$ are positive continuous functions of $z \in \mathbb{C}^{n}, j \in\{1,2, \ldots, n\}$. An entire function, $F(z), z \in \mathbb{C}^{n}$, is called a function of bounded $\mathbf{L}$ index in joint variables [1] if there exists a number $m \in \mathbb{Z}_{+}$ such that for all $z \in \mathbb{C}^{n}$ and $J=\left(j_{1}, j_{2}, \ldots, j_{n}\right) \in \mathbb{Z}_{+}^{n}$

$$
\frac{\left|F^{(J)}(z)\right|}{J ! \mathbf{L}^{J}(z)} \leq \max \left\{\frac{\left|F^{(K)}(z)\right|}{K ! \mathbf{L}^{K}(z)}: K \in \mathbb{Z}_{+}^{n},\|K\| \leq m\right\} .
$$

If $l_{j}=l_{j}\left(\left|z_{j}\right|\right)$ then we obtain a concept of entire functions of bounded L-index in a sense of definition given in [24]. If $l_{j}\left(z_{j}\right) \equiv 1, j \in\{1,2, \ldots, n\}$, then the entire function is called a function of bounded index in joint variables [4-8, 25].

The least integer $m$ for which inequality (3) holds is called $\mathbf{L}$-index in joint variables of the function $F$ and is denoted by $N(F, \mathbf{L})$.

For $R \in \mathbb{R}_{+}^{n}, j \in\{1, \ldots, n\}$ and $\mathbf{L}(z)=\left(l_{1}(z), \ldots, l_{n}(z)\right)$ we define

$$
\begin{aligned}
& \lambda_{1, j}(R)=\inf _{z^{0} \in \mathbb{C}^{n}} \inf \left\{\frac{l_{j}(z)}{l_{j}\left(z^{0}\right)}: z \in \mathbb{D}^{n}\left[z^{0}, \frac{R}{\mathbf{L}\left(z^{0}\right)}\right]\right\}, \\
& \lambda_{2, j}(R)=\sup _{z^{0} \in \mathbb{C}^{n}} \sup \left\{\frac{l_{j}(z)}{l_{j}\left(z^{0}\right)}: z \in \mathbb{D}^{n}\left[z^{0}, \frac{R}{\mathbf{L}\left(z^{0}\right)}\right]\right\}, \\
& \Lambda_{1}(R)=\left(\lambda_{1, j}(R), \ldots, \lambda_{1, n}(R)\right), \\
& \Lambda_{2}(R)=\left(\lambda_{2,1}(R), \ldots, \lambda_{2, n}(R)\right) .
\end{aligned}
$$

By $Q^{n}$ we denote a class of functions $\mathbf{L}(z)$ which for every $R \in \mathbb{R}_{+}^{n}$ and $j \in\{1, \ldots, n\}$ satisfy the condition

$$
0<\lambda_{1, j}(R) \leq \lambda_{2, j}(R)<+\infty
$$

If $n=1$ then $Q \equiv Q^{1}$.

Let $\widetilde{\mathbf{L}}(z)=\left(\widetilde{l}_{1}(z), \ldots, \widetilde{l}_{n}(z)\right)$. A notation $\mathbf{L} \asymp \widetilde{\mathbf{L}}$ means that there exist $\Theta_{1}=\left(\theta_{1, j}, \ldots, \theta_{1, n}\right) \in \mathbb{R}_{+}^{n}, \Theta_{2}=\left(\theta_{2, j}, \ldots, \theta_{2, n}\right) \in$ $\mathbb{R}_{+}^{n}$ such that $\forall z \in \mathbb{C}^{n} \theta_{1, j} \widetilde{l}_{j}(z) \leq l_{j}(z) \leq \theta_{2, j} \widetilde{l}_{j}(z)$.

\section{Auxiliary Propositions}

We need the following theorems.

Theorem 1 ([11, p. 158, Th. 4.2], see also [23]). Let $\mathbf{L} \in Q^{n}$ and $\mathbf{L}=\widetilde{\mathbf{L}}$. An entire function $F: \mathbb{C}^{n} \rightarrow \mathbb{C}$ has bounded $\tilde{\mathbf{L}}$-index in joint variables if and only if $F$ has bounded $\mathbf{L}$-index in joint variables.

Theorem 2 (see [1]). Let $\mathbf{L} \in Q^{n}$. An entire function $F$ is of bounded $\mathbf{L}$-index in joint variables if and only if, for any $R^{\prime}$, 
$R^{\prime \prime}, \mathbf{0}<R^{\prime}<R^{\prime \prime}$, there exists a number $p_{1}=p_{1}\left(R^{\prime}, R^{\prime \prime}\right) \geq 1$ such that for every $z^{0} \in \mathbb{C}^{n}$ inequality

$$
\begin{aligned}
& \max \left\{|F(z)|: z \in \mathbb{T}^{n}\left(z^{0}, \frac{R^{\prime \prime}}{\mathbf{L}\left(z^{0}\right)}\right)\right\} \\
& \quad \leq p_{1} \max \left\{|F(z)|: z \in \mathbb{T}^{n}\left(z^{0}, \frac{R^{\prime}}{\mathbf{L}\left(z^{0}\right)}\right)\right\} .
\end{aligned}
$$

holds.

Remark 3. It was also proved that the condition "for any $R^{\prime}$, $R^{\prime \prime}, \mathbf{0}<R^{\prime}<R^{\prime \prime}$, there exists a number $p_{1}=p_{1}\left(R^{\prime}, R^{\prime \prime}\right) \geq 1$ " in Theorem 2 can be replaced by the condition "there exist $R^{\prime}, R^{\prime \prime}, \mathbf{0}<R^{\prime}<\mathbf{1}<R^{\prime \prime}$, and $p_{1}=p_{1}\left(R^{\prime}, R^{\prime \prime}\right) \geq 1$ ". It is Theorem 5 in [1].

Now we relax the restriction $R^{\prime}<\mathbf{1}<R^{\prime \prime}$ in sufficient conditions.

Theorem 4. Let $\mathbf{L} \in Q^{n}, F: \mathbb{C} \rightarrow \mathbb{C}^{n}$ be an entire function. If there exist $R^{\prime}, R^{\prime \prime}, \mathbf{0}<R^{\prime}<R^{\prime \prime}$, and $p_{1}=p_{1}\left(R^{\prime}, R^{\prime \prime}\right) \geq 1$ such that for every $z^{0} \in \mathbb{C}^{n}$ inequality (6) holds; then the function $F$ has bounded $\mathbf{L}$-index in joint variables.

Proof. From (6) with $\mathbf{0}<R^{\prime}<R^{\prime \prime}$ it follows that

$$
\begin{aligned}
& \max \left\{|F(z)|: z \in \mathbb{T}^{n}\left(z^{0}, \frac{2 R^{\prime \prime}}{R^{\prime}+R^{\prime \prime}} \frac{R^{\prime}+R^{\prime \prime}}{2 \mathbf{L}\left(z^{0}\right)}\right)\right\} \\
& \leq P_{1} \max \left\{|F(z)|: z \in \mathbb{T}^{n}\left(z^{0}, \frac{2 R^{\prime}}{R^{\prime}+R^{\prime \prime}} \frac{R^{\prime}+R^{\prime \prime}}{2 \mathbf{L}\left(z^{0}\right)}\right)\right\} .
\end{aligned}
$$

Denoting $\widetilde{\mathbf{L}}(z)=2 \mathbf{L}(z) /\left(R^{\prime}+R^{\prime \prime}\right)$, we obtain

$$
\begin{aligned}
& \max \left\{|F(z)|: z \in \mathbb{T}^{n}\left(z^{0}, \frac{2 R^{\prime \prime}}{\left(R^{\prime}+R^{\prime \prime}\right) \tilde{\mathbf{L}}\left(z^{0}\right)}\right)\right\} \\
& \leq P_{1} \max \left\{|F(z)|: z \in \mathbb{T}^{n}\left(z^{0}, \frac{2 R^{\prime}}{\left(R^{\prime}+R^{\prime \prime}\right) \tilde{\mathbf{L}}\left(z^{0}\right)}\right)\right\},
\end{aligned}
$$

where $\mathbf{0}<2 R^{\prime} /\left(R^{\prime}+R^{\prime \prime}\right)<\mathbf{1}<2 R^{\prime \prime} /\left(R^{\prime}+R^{\prime \prime}\right)$. In view of Remark 3, $F$ has bounded $\widetilde{\mathbf{L}}$-index in joint variables. By Theorem 1, the function $F$ is bounded L-index in joint variables.

Note that Theorem 4 is new even if $\mathbf{L}(z) \equiv \mathbf{1}$.

Lemma 5. If $L: \mathbb{C}^{n} \rightarrow \mathbb{R}_{+}$is a continuous function such that $\left(\forall R \in \mathbb{R}_{+}^{n}\right) \Lambda_{2}(R)<\infty$ then $\left(\forall R \in \mathbb{R}_{+}^{n}\right) \Lambda_{1}(R) \geq$ $\mathbf{1} / \Lambda_{2}\left(R \Lambda_{2}(R)\right)>0$.

Proof. Let $\left(\forall R \in \mathbb{R}_{+}^{n}\right) \Lambda_{2}(R)<\infty$ i.e. $\forall j \in\{1, \ldots$, n\} $\lambda_{2, j}(R)<+\infty$. Hence, we have $l_{j}(z) \leq \lambda_{2, j}(R) l_{j}\left(z^{0}\right)$ for $z \in D^{n}\left(z^{0}, R / \mathbf{L}\left(z^{0}\right)\right)$. This means that $\left|z_{j}-z_{j}^{0}\right| \leq r_{j} / l_{j}\left(z^{0}\right) \leq$ $r_{j} \lambda_{2, j}(R) / l_{j}(z)$. Using definition of $\lambda_{1, j}(R)$, we deduce

$$
\begin{aligned}
& \inf \left\{\frac{l_{j}(z)}{l_{j}\left(z^{0}\right)}: z \in \mathbb{D}^{n}\left(z^{0}, \frac{R}{\mathbf{L}\left(z^{0}\right)}\right)\right\} \\
& =\frac{1}{\sup \left\{l_{j}\left(z^{0}\right) / l_{j}(z): z \in \mathbb{D}^{n}\left(z^{0}, R / \mathbf{L}\left(z^{0}\right)\right)\right\}} \\
& \geq \frac{1}{\sup \left\{l_{j}\left(z^{0}\right) / l_{j}(z):\left|z_{j}^{0}-z_{j}\right| \leq r_{j} \lambda_{2, j}(R) / l_{j}(z), j \in\{1, \ldots, n\}\right\}} \\
& \geq \frac{1}{\lambda_{2, j}\left(R \Lambda_{2}(R)\right)} .
\end{aligned}
$$

Thus, $\lambda_{1, j}(R) \geq 1 / \lambda_{2, j}\left(R \Lambda_{2}(R)\right)$.

Remark 6. By Lemma 5 the left inequality in (5) is excessive because the condition $\lambda_{2, j}(R)<+\infty$ implies $\lambda_{1, j}(R)>0$. But in our considerations we will use so $\Lambda_{1}(R)$ as $\Lambda_{2}(R)$. It is convenient.

\section{Estimate Maximum Modulus on a Skeleton in Polydisc}

Let $Z_{F}$ be a zero set of entire function $F$. We denote

$$
\begin{gathered}
G_{R}(F)=\bigcup_{z^{0} \in Z_{F}}\left\{z \in \mathbb{C}^{n}:\left|z_{j}-z_{j}^{0}\right|<\frac{r_{j}}{l_{j}\left(z^{0}\right)} \forall j\right. \\
\quad \in\{1,2, \ldots, n\}\}=\bigcup_{z^{0} \in Z_{F}} \mathbb{D}^{n}\left(z^{0}, \frac{R}{\mathbf{L}\left(z^{0}\right)}\right) .
\end{gathered}
$$

Theorem 7. Let $\mathbf{L} \in Q^{n}, F$ be an entire in $\mathbb{C}^{n}$ function. If $\exists R>$ $\mathbf{0} \exists p_{2} \geq 1 \exists \Theta \in \mathbb{R}_{+}^{n}, \mathbf{0}<\Theta<R, \exists R^{\prime}>\mathbf{0},\left(R^{\prime}=\mathbf{0}\right.$ for $\left.Z_{F}=\emptyset\right)$ such that $\forall z^{0} \in \mathbb{C}^{n} \exists R^{0}=R^{0}\left(z^{0}\right) \in \mathbb{R}_{+}^{n}, \Theta \leq R^{0} \leq R$, for which

$$
\begin{aligned}
& \operatorname{meas}\left\{\mathbb{T}^{n}\left(z^{0}, \frac{R^{0}}{\mathbf{L}\left(z^{0}\right)}\right) \cap G_{R^{\prime}}(F)\right\} \\
& <\left(\frac{2 \pi}{3}\right)^{n} \prod_{j=1}^{n} \frac{\theta_{j}}{\lambda_{2, j}(2(R+\mathbf{1})) l_{j}\left(z^{0}\right)}, \\
& \max \left\{|F(z)|: z \in \mathbb{T}^{n}\left(z^{0}, \frac{R^{0}}{\mathbf{L}\left(z^{0}\right)}\right)\right\} \\
& \quad \leq p_{2} \min \left\{|F(z)|: z \in \mathbb{T}^{n}\left(z^{0}, \frac{R^{0}}{\mathbf{L}\left(z^{0}\right)}\right) \backslash G_{R^{\prime}}(F)\right\}
\end{aligned}
$$

then the function $F$ has bounded L-index in joint variables (meas is the Lebesgue measure on the skeleton in the polydisc).

Proof. By Theorem 4, we will show that $\exists p_{1}>0 \forall z^{0} \in \mathbb{C}^{n}$

$$
\begin{aligned}
& \max \left\{|F(z)|: z \in \mathbb{T}^{n}\left(z^{0}, \frac{R+\mathbf{1}}{\mathbf{L}\left(z^{0}\right)}\right)\right\} \\
& \quad \leq p_{1} \max \left\{|F(z)|: z \in \mathbb{T}^{n}\left(z^{0}, \frac{R}{\mathbf{L}\left(z^{0}\right)}\right)\right\} .
\end{aligned}
$$


Denote $l_{j}^{*}=\max \left\{l_{j}(z): z \in \mathbb{D}^{n}\left[z^{0}, 2(R+\mathbf{1}) / \mathbf{L}\left(z^{0}\right)\right]\right\}, \rho_{j, 0}=$ $r_{j} / l_{j}\left(z^{0}\right), \rho_{j, k}=\rho_{j, 0}+\left(k \cdot \theta_{j}\right) / l_{j}^{*}, k \in \mathbb{N}, j \in\{1, \ldots, n\}$. The following estimate holds

$$
\frac{\theta_{j}}{l_{j}^{*}}<\frac{r_{j}}{l_{j}^{*}} \leq \frac{r_{j}}{l_{j}\left(z^{0}\right)}<\frac{2 r_{j}+2}{l_{j}\left(z^{0}\right)}-\frac{r_{j}+1}{l_{j}\left(z^{0}\right)} .
$$

Hence, there exists $S^{*}=\left(s_{1}^{*}, \ldots, s_{n}^{*}\right) \in \mathbb{N}$ independent of $z^{0}$ such that

$$
\rho_{j, m_{j}-1}<\frac{r_{j}+1}{l_{j}\left(z^{0}\right)}<\rho_{j, m_{j}} \leq \frac{2 r_{j}+2}{l_{j}\left(z^{0}\right)}
$$

for some $m_{j}=m_{j}\left(z^{0}\right) \leq s_{j}^{*}$ because $\mathbf{L} \in Q^{n}$. Indeed,

$$
\begin{aligned}
& \frac{\left(\left(2 r_{j}+2\right) / l_{j}\left(z^{0}\right)-\rho_{j, 0}\right)}{\theta_{j} / l_{j}^{*}}=\left(2 r_{j}+2-r_{j}\right) \frac{l_{j}^{*}}{\theta_{j} l_{j}\left(z^{0}\right)} \\
& =\frac{r_{j}+2}{\theta_{j}} \max \left\{\frac{l_{j}(z)}{l_{j}\left(z^{0}\right)}: z \in \mathbb{D}^{n}\left[z^{0}, \frac{2(R+\mathbf{1})}{\mathbf{L}\left(z^{0}\right)}\right]\right\} \\
& \leq \frac{r_{j}+2}{\theta_{j}} \lambda_{2, j}(2(R+\mathbf{1})) .
\end{aligned}
$$

Thus, $s_{j}^{*}=\left[\left(\left(r_{j}+2\right) / \theta_{j}\right) \lambda_{2, j}(2(R+\mathbf{1}))\right]$, where $[x]$ is the integer part of $x \in \mathbb{R}$.

Let $M_{0}=\left(m_{1}, \ldots, m_{n}\right)$ and $\tau_{K}^{* *}$ be such a point in $\mathbb{C}^{n}$ that

$$
\left|F\left(\tau_{K}^{* *}\right)\right|=\max \left\{|F(z)|: z \in \mathbb{T}^{n}\left(z^{0}, \mathscr{R}_{K}\right)\right\}
$$

where $K=\left(k_{1}, \ldots, k_{n}\right), \mathscr{R}_{K}=\left(\rho_{1, k_{1}}, \ldots, \rho_{n, k_{n}}\right)$ and $\tau_{j, K}^{*}$ be the intersection point in $\mathbb{C}$ of the segment $\left[z_{j}^{0}, \tau_{j, K}^{* *}\right]$ with $\left|z_{j}-z_{j}^{0}\right|=$ $\rho_{j, k_{j}-1}$. We construct a sequence of polydisc $\mathbb{D}^{n}\left(z^{0}, \mathscr{R}_{K}\right)$ with $K \leq M_{0}, \mathscr{R}_{0}=R / \mathbf{L}\left(z^{0}\right)=\left(\rho_{1,0}, \ldots, \rho_{n, 0}\right)$ and $\Theta / \mathbf{L}\left(z^{0}\right)=$ $\left(\theta_{1} / l_{1}^{*}, \ldots, \theta_{n} / l_{n}^{*}\right)$ (see Figures 1 and 2$)$.

Denote $\alpha_{K}^{(j)}=\left(\tau_{1, K}^{* *}, \ldots, \tau_{j-1, K}^{* *}, \tau_{j, K}^{*}, \tau_{j+1, K}^{* *}, \ldots, \tau_{n, K}^{* *}\right)$. Hence, for every $r_{j}>\theta_{j}$ and $K \leq S^{*}:\left|\tau_{j, K}^{*}-\tau_{j, K}^{* *}\right|=\theta_{j} / l_{j}^{*} \leq$ $r_{j} / l_{j}\left(\alpha_{K}^{(j)}\right)$. Thus, for some $R^{0}=R^{0}\left(\alpha_{K}^{(j)}\right) \in \mathbb{R}_{+}^{n}, \Theta \leq R^{0} \leq R$, we deduce

$$
\begin{aligned}
& \left|F\left(\tau_{K}^{* *}\right)\right| \leq \max \left\{|F(z)|: z \in \mathbb{T}^{n}\left(\alpha_{K}^{(j)}, \frac{R^{0}}{\mathbf{L}\left(\alpha_{K}^{(j)}\right)}\right)\right\} \\
& \quad \leq p_{2} \min \{|F(z)|: z \\
& \left.\quad \in \mathbb{T}^{n}\left(\alpha_{K}^{(j)}, \frac{R^{0}}{\mathrm{~L}\left(\alpha_{K}^{(j)}\right)}\right) \backslash G_{R^{\prime}}(F)\right\} \leq p_{2}
\end{aligned}
$$

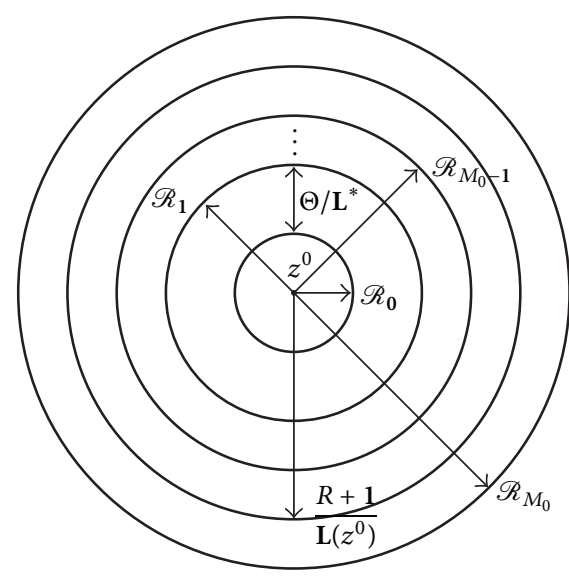

Figure 1

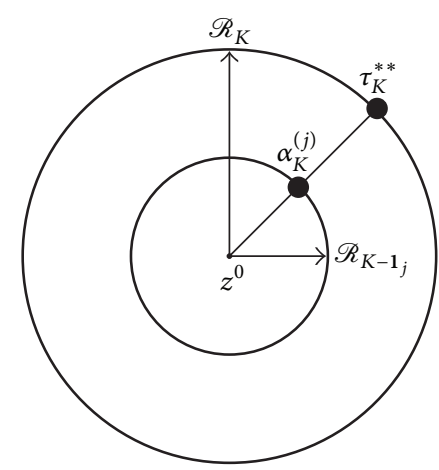

Figure 2

$$
\begin{aligned}
& \cdot \min \{|F(z)|: z \\
& \in \mathbb{T}^{n}\left(\alpha_{K}^{(j)}, \frac{R^{0}}{\mathbf{L}\left(\alpha_{K}^{(j)}\right)}\right) \backslash G_{R^{\prime}}(F), z \\
& \left.\in \mathbb{D}^{n}\left[z^{0}, \mathscr{R}_{K-\mathbf{1}_{j}}\right]\right\} \leq p_{2} \max \{|F(z)|: z \\
& \left.\in \mathbb{T}^{n}\left(z^{0}, \mathscr{R}_{K-\mathbf{1}_{j}}\right)\right\} .
\end{aligned}
$$

To deduce (18), we implicitly used that

$$
\begin{aligned}
& \left(\mathbb{T}^{n}\left(\alpha_{K}^{(j)}, \frac{R^{0}}{\mathbf{L}\left(\alpha_{K}^{(j)}\right)}\right) \backslash G_{R^{\prime}}(F)\right) \cap \mathbb{D}^{n}\left[z^{0}, \mathscr{R}_{K-\mathbf{1}_{j}}\right] \\
& \quad \neq \emptyset .
\end{aligned}
$$

Condition (11) provides (19). Indeed, we will find a lower estimate of measure of the set $\mathbb{T}^{n}\left(\alpha_{K}^{(j)}, R^{0} / \mathbf{L}\left(\alpha_{K}^{(j)}\right)\right) \cap$ $\mathbb{D}^{n}\left[z^{0}, \mathscr{R}_{K-\mathbf{1}_{j}}\right]$ and will show that the measure is not lesser than a left part of inequality (11). 


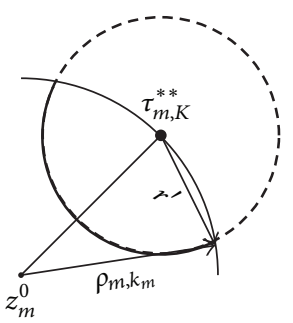

Figure 3: With $r^{\prime}=r_{m}^{0} / l_{m}\left(\alpha_{K}^{(j)}\right)$.

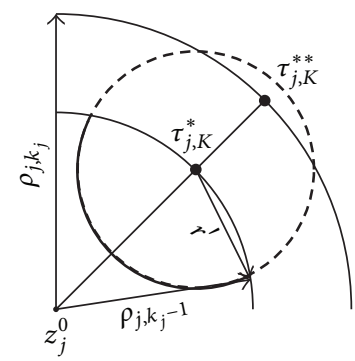

FIGURE 4: With $r^{\prime}=r_{j}^{0} / l_{j}\left(\alpha_{K}^{(j)}\right)$.

The set $\mathbb{T}^{n}\left(\alpha_{K}^{(j)}, R^{0} / \mathbf{L}\left(\alpha_{K}^{(j)}\right)\right) \cap \mathbb{D}^{n}\left[z^{0}, \mathscr{R}_{K-\mathbf{1}_{j}}\right]$ is a Cartesian product of the following arcs on circles: for every $m \in$ $\{1, \ldots, n\}, m \neq j$ (see Figure 3 )

$$
\begin{gathered}
\left\{z_{m} \in \mathbb{C}:\left|z_{m}-\tau_{m, K}^{* *}\right|=\frac{r_{m}^{0}}{l_{m}\left(\alpha_{K}^{(j)}\right)}\right\} \\
\cap\left\{z_{m} \in \mathbb{C}:\left|z_{m}-z_{m}^{0}\right| \leq \rho_{m, k_{m}}\right\}
\end{gathered}
$$

and for $m=j$ (see Figure 4)

$$
\begin{array}{r}
\left\{z_{j} \in \mathbb{C}:\left|z_{j}-\tau_{j, K}^{*}\right|=\frac{r_{j}^{0}}{l_{j}\left(\alpha_{K}^{(j)}\right)}\right\} \\
\cap\left\{z_{j} \in \mathbb{C}:\left|z_{j}-z_{j}^{0}\right| \leq \rho_{j, k_{j}-1}\right\} .
\end{array}
$$

It is easy to prove that the length of arc equals

$$
\begin{array}{ll}
\frac{2 r_{m}^{0}}{l_{m}\left(\alpha_{K}^{(j)}\right)} \cdot \arccos \frac{r_{m}^{0}}{2 l_{m}\left(\alpha_{K}^{(j)}\right) \rho_{m, k_{m}}} & \text { for } m \neq j, \\
\frac{2 r_{j}^{0}}{l_{j}\left(\alpha_{K}^{(j)}\right)} \cdot \arccos \frac{r_{j}^{0}}{2 l_{j}\left(\alpha_{K}^{(j)}\right) \rho_{j, k_{j}-1}} & \text { for } m=j .
\end{array}
$$

But for $m \neq j r_{m}^{0} / l_{m}\left(\alpha_{K}^{(j)}\right) \leq \rho_{m, k_{m}}$ and $r_{j}^{0} / l_{j}\left(\alpha_{K}^{(j)}\right) \leq$ $\rho_{j, k_{j}-1}$ the argument in arccosine from (23) and (22) does not exceed $1 / 2$. This means that the length of arc is not lesser than

$$
\begin{aligned}
& \frac{2 r_{m}^{0}}{l_{m}\left(\alpha_{K}^{(j)}\right)} \arccos \frac{1}{2} \geq \frac{2 \theta_{m} \pi}{3 l_{m}\left(z^{0}\right) \lambda_{2, m}(2(R+\mathbf{1}))} \\
& \text { for every } m \in\{1,2, \ldots, n\},
\end{aligned}
$$

because $\mathbf{L} \in Q^{n}$. Accordingly, the measure of the set

$$
\mathbb{T}^{n}\left(\alpha_{K}^{(j)}, \frac{R^{0}}{\mathbf{L}\left(\alpha_{K}^{(j)}\right)}\right) \cap \mathbb{D}^{n}\left[z^{0}, \mathscr{R}_{K-\mathbf{1}_{j}}\right]
$$

on the skeleton of polydisc is always not lesser than $\prod_{m=1}^{n}\left(2 \theta_{m} \pi / 3 l_{m}\left(z^{0}\right) \lambda_{2, m}(2(R+1))\right)$. Assuming a strict inequality in (11), we deduce that (19) is valid.

Applying (18) $m_{j}$ th times in every variable $z_{j}$, we obtain

$$
\begin{aligned}
& \max \left\{|F(z)|: z \in \mathbb{T}^{n}\left(z^{0}, \frac{R+\mathbf{1}}{\mathbf{L}\left(z^{0}\right)}\right)\right\} \\
& \leq \max \left\{|F(z)|: z \in \mathbb{T}^{n}\left(z^{0}, \mathscr{R}_{M_{0}}\right)\right\} \leq p_{2} \\
& \cdot \max \left\{|F(z)|: z \in \mathbb{T}^{n}\left(z^{0}, \mathscr{R}_{M_{0}-\mathbf{1}_{n}}\right)\right\} \leq p_{2}^{m_{n}} \\
& \cdot \max \left\{|F(z)|: z \in \mathbb{T}^{n}\left(z^{0}, \mathscr{R}_{M_{0}-m_{n} \mathbf{1}_{n}}\right)\right\} \leq \cdots \\
& \leq p_{2}^{m_{n}+1} \\
& \cdot \max \left\{|F(z)|: z \in \mathbb{T}^{n}\left(z^{0}, \mathscr{R}_{M_{0}-m_{n} \mathbf{1}_{n}-\mathbf{1}_{n-1}}\right)\right\} \\
& \leq p_{2}^{m_{n}+m_{n-1}} \\
& \cdot \max \left\{|F(z)|: z \in \mathbb{T}^{n}\left(z^{0}, \mathscr{R}_{M_{0}-m_{n} \mathbf{1}_{n}-m_{n-1} \mathbf{1}_{n-1}}\right)\right\} \\
& \leq \cdots \leq p_{2}^{\left\|M_{0}\right\|} \max \left\{|F(z)|: z \in \mathbb{T}^{n}\left(z^{0}, \mathscr{R}_{\mathbf{0}}\right)\right\} \\
& \leq p_{2}^{\left\|S^{*}\right\|} \max \left\{|F(z)|: z \in \mathbb{T}^{n}\left(z^{0}, \frac{R}{\mathbf{L}\left(z^{0}\right)}\right)\right\} \text {. }
\end{aligned}
$$

By Theorem 2 the function $F$ has bounded $\mathbf{L}$-index in joint variables.

Let us denote $c\left(z^{\prime}, r\right)=\left\{z \in \mathbb{C}:\left|z-z^{\prime}\right|=r / l\left(z^{\prime}\right)\right\}$. For $n=1$ Theorem 7 implies the following corollary.

Corollary 8. Let $l \in Q, f$ be an entire function. If $\exists r>$ $0, \exists r^{\prime} \geq 0, \exists p_{2} \geq 1 \exists \theta \in(0, r)$, such that $\forall z^{0} \in$ $\mathbb{C} \exists r^{0}=r^{0}\left(z^{0}\right) \in[\theta ; r]$, and meas $\left\{c\left(z^{0}, r^{0}\right) \cap G_{R^{\prime}}(F)\right\}<$ $2 \pi \theta / 3 l\left(z^{0}\right) \lambda_{2}(2 r+2)$ and

$$
\begin{aligned}
& \max \left\{|f(z)|: z \in c\left(z^{0}, r^{0}\right)\right\} \\
& \quad \leq p_{2} \min \left\{|f(z)|: z \in c\left(z^{0}, r^{0}\right) \backslash G_{r^{\prime}}(f)\right\}
\end{aligned}
$$

then the function $f$ has bounded l-index (here meas means the Lebesgue measure on the circle). 
In a some sense, this corollary is new even for an entire function of one variable because the circle $c\left(z^{0}, r^{0}\right)$ can contain zeros of the function $f$. Meanwhile, in corresponding theorem from $[26,27]$ the circle $c\left(z^{0}, r^{0}\right)$ is chosen such that $f(z) \neq 0$ for all $z \in c\left(z^{0}, r^{0}\right)$.

\section{Behavior of Partial Logarithmic Derivatives}

Denote $\mathcal{F}=\left\{\left(j_{1}, \ldots, j_{n}\right): j_{i} \in\{0,1\}, i \in\{1, \ldots, n\}\right\} \backslash \mathbf{0}$.

Theorem 9. Let $\mathbf{L} \in Q^{n}$. If an entire function $F$ satisfies the following conditions

(1) for every $R>\mathbf{0}$ there exists $p_{1}=p_{1}(R)>0$ such that for all $z \in \mathbb{C}^{n} \backslash G_{R}(F)$ and for all $J \in \mathcal{J}$

$$
\left|(\ln F(z))^{(J)}\right| \leq p_{1} \mathbf{L}^{J}(z),
$$

where $\ln F(z)$ is the principal value of logarithm.

(2) for every $R>\mathbf{0}$ and $R^{\prime} \geq \mathbf{0}$ exists $p_{2}=$ $p_{2}\left(R, R^{\prime}\right) \geq 1$ that for all $z^{0} \in \mathbb{C}^{n}$ such that $T^{n}\left(z^{0}, R / \mathbb{L}\left(z^{0}\right)\right) \backslash G_{R^{\prime}}(F)=\bigcup_{i} C_{i} \neq \emptyset$, where the sets $C_{i}$ are connected disjoint sets, and either (a) $\max _{i} \min _{z \in C_{i}}|F(z)| \leq p_{2} \min _{i} \min _{z \in C_{i}}|F(z)|$, or (b) $\max _{i} \max _{z \in C_{i}}|F(z)| \leq p_{2} \min _{i} \max _{z \in C_{i}}|F(z)|$, or (c) $\left|F\left(z^{*}\right)\right|=\max _{i} \max _{z \in C_{i}}|F(z)|,\left|F\left(z^{* *}\right)\right|=$ $\min _{i} \min _{z \in C_{i}}|F(z)|$, and $z^{*}, z^{* *}$ belong to the same set $C_{i_{0}}$

(3) for every $R>0$ there exists $n^{*}(R)>0$ such that for all $z \in \mathbb{C}^{n}$

$$
\text { meas }\left\{Z_{F} \cap \mathbb{D}^{n}\left[z, \frac{R}{\mathbf{L}(z)}\right]\right\} \leq n^{*}(R) \text {. }
$$

then $F$ has bounded $\mathbf{L}$-index in joint variables (here meas is $(2 n-2)$-dimensional of the Lebesgue measure).

Proof. Let $z^{0} \in \mathbb{C}^{n}$ be arbitrarily chosen point. In view of Theorem 7 we need to prove that

$$
\begin{aligned}
& \text { meas }\left\{\mathbb{T}^{n}\left(z^{0}, \frac{R^{0}}{\mathbf{L}\left(z^{0}\right)}\right) \cap G_{R^{\prime}}(F)\right\} \\
& <\left(\frac{2 \pi}{3}\right)^{n} \prod_{j=1}^{n} \frac{\theta_{j}}{\lambda_{2, j}(2(R+\mathbf{1})) l_{j}\left(z^{0}\right)} \\
& \quad \text { for some } R^{0}=R^{0}\left(z^{0}\right) .
\end{aligned}
$$

Let $R>\mathbf{0}$ be arbitrary radius. We choose $\Theta, R^{\prime} \in \mathbb{R}_{+}^{n}$ such that $\theta_{j}<2 r_{j} /\left(2+3 \lambda_{2, j}(2(R+\mathbf{1}))\right)$,

$$
\begin{aligned}
& r_{j}^{\prime}<\min \left\{\theta_{j},\right. \\
&\left.\sqrt{\frac{2 \lambda_{1, j}^{2}\left(R+R \Lambda_{2}(R) / \Lambda_{1}(R)\right) \theta_{j}\left(r_{j}-\theta_{j}\right)}{3\left(n^{*}\left(R+R \Lambda_{2}(R) / \Lambda_{1}(R)\right)\right)^{1 / n} \lambda_{2, j}(2(R+1))}}\right\}, \\
& j \in\{1, \ldots, n\} .
\end{aligned}
$$

Let $d S=d s_{1} \cdot \ldots \cdot d s_{n}, S=\left(s_{1}, \ldots, s_{n}\right), \omega_{z}$ be a volume measure in $\mathbb{R}^{2 n}$. Clearly, (see [28, p. 75-76])

$$
\begin{aligned}
& \int_{\mathbb{D}^{n}\left(z^{0}, R\right)} u(z) d \omega_{z}=\int_{0}^{r_{1}} \cdots \int_{0}^{r_{n}} s_{1} \cdots s_{n} \\
& \cdot\left(\int_{0}^{2 \pi} \cdots \int_{0}^{2 \pi} u\left(z^{0}+S e^{i \Theta}\right) d \theta_{1} \cdots d \theta_{n}\right) d s_{1} \cdots d s_{n} \\
& \quad=\int_{0}^{R}\left(\int_{0}^{2 \pi} \cdots \int_{0}^{2 \pi} u\left(z^{0}+S e^{i \Theta}\right) d\left(s_{1} \theta_{1}\right) \cdots d\left(s_{n} \theta_{n}\right)\right) d S,
\end{aligned}
$$

where $u$ is plurisubharmonic function. Hence,

$$
\begin{aligned}
& \int_{0}^{R / \mathbf{L}\left(z^{0}\right)} \operatorname{meas}\left\{\mathbb{T}^{n}\left(z^{0}, S\right) \cap G_{R^{\prime}}(F)\right\} d S \\
& \quad=\operatorname{meas}\left\{\mathbb{D}^{n}\left[z^{0}, \frac{R}{\mathbf{L}\left(z^{0}\right)}\right] \cap G_{R^{\prime}}(F)\right\} .
\end{aligned}
$$

Obviously, there can exist points $z^{\prime} \in Z_{F} \backslash \mathbb{D}^{n}\left[z^{0}, R / \mathbf{L}\left(z^{0}\right)\right]$ such that

$$
\mathbb{D}^{n}\left[z^{0}, \frac{R}{\mathbf{L}\left(z^{0}\right)}\right] \cap \mathbb{D}^{n}\left[z^{\prime}, \frac{R^{\prime}}{\mathbf{L}\left(z^{\prime}\right)}\right] \neq \emptyset .
$$

Let $z_{j}^{\prime \prime}$ be the intersection point of the segment $\left[z_{j}^{0}, z_{j}^{\prime}\right]$ and the circle $\left|z_{j}-z_{j}^{0}\right|=r_{j} / l_{j}\left(z^{0}\right), j \in\{1, \ldots, n\}$. Then $\left|z_{j}^{\prime \prime}-z_{j}^{\prime}\right| \leq$ $r_{j}^{\prime} / l_{j}\left(z^{\prime}\right)$ and $z^{\prime \prime} \in \mathbb{T}^{n}\left(z^{0}, R / \mathbf{L}\left(z^{0}\right)\right)$. Using $\mathbf{L} \in Q^{n}$, we estimate maximum distance between $z_{j}^{0}$ and $z_{j}^{\prime}$ :

$$
\begin{aligned}
l_{j}\left(z^{\prime}\right) & \geq \frac{l_{j}\left(z^{\prime}\right)}{l_{j}\left(z^{\prime \prime}\right)} \cdot \frac{l_{j}\left(z^{\prime \prime}\right)}{l_{j}\left(z^{0}\right)} \cdot l_{j}\left(z^{0}\right) \\
& \geq \frac{\lambda_{1, j}(R)}{\lambda_{2, j}\left(R^{\prime}\right)} \cdot l_{j}\left(z^{0}\right) \geq \frac{\lambda_{1, j}(R)}{\lambda_{2, j}\left(R^{\prime}\right)} l_{j}\left(z^{0}\right), \\
\left|z_{j}^{0}-z_{j}^{\prime}\right| & \leq\left|z^{0}-z_{j}^{\prime \prime}\right|+\left|z_{j}^{\prime \prime}-z_{j}^{\prime}\right| \leq \frac{r_{j}}{l_{j}\left(z^{0}\right)}+\frac{r_{j}^{\prime}}{l_{j}\left(z_{j}^{\prime}\right)} \\
& \leq \frac{r_{j}}{l_{j}\left(z^{0}\right)}\left(1+\frac{\lambda_{2, j}(R)}{\lambda_{1, j}\left(R^{\prime}\right)}\right) .
\end{aligned}
$$

Denote $R^{\prime \prime}=R+R \Lambda_{2}(R) / \Lambda_{1}(R)$. Let $V_{2 n-2}$ be a $(2 n-$ 2)-dimensional volume, $\chi_{F}(z)$ a characteristic function of zero set of the function $F$. Now we replace the measure in (33) by integrating on zero set in polydisc $\mathbb{D}^{n}\left[z^{0}, R^{\prime \prime}\right.$, $\mathbf{L}\left(z^{0}\right)$ ]: 


$$
\begin{aligned}
& \int_{0}^{R / \mathbf{L}\left(z^{0}\right)} \operatorname{meas}\left\{\mathbb{T}^{n}\left(z^{0}, S\right) \cap G_{R^{\prime}}(F)\right\} d S \leq \int_{Z_{F} \cap \mathbb{D}^{n}\left[z^{0}, R^{\prime \prime} / \mathbf{L}\left(z^{0}\right)\right]} \chi_{F}(z) \pi^{n} \prod_{j=1}^{n} \frac{\left(r_{j}^{\prime}\right)^{2}}{l_{j}^{2}(z)} d V_{2 n-2} \\
& \quad \leq \pi^{n} \prod_{j=1}^{n} \frac{\left(r_{j}^{\prime}\right)^{2}}{\lambda_{1, j}^{2}\left(R^{\prime \prime}\right) l_{j}^{2}\left(z^{0}\right)} \int_{Z_{F} \cap \mathbb{D}^{n}\left[z^{0}, R^{\prime \prime} / \mathbf{L}\left(z^{0}\right)\right]} \chi_{F}(z) d V_{2 n-2} \leq n^{*}\left(R^{\prime \prime}\right) \pi^{n} \prod_{j=1}^{n} \frac{\left(r_{j}^{\prime}\right)^{2}}{\lambda_{1, j}^{2}\left(R^{\prime \prime}\right) l_{j}^{2}\left(z^{0}\right)} \\
& \quad<n^{*}\left(R^{\prime \prime}\right) \pi^{n} \prod_{j=1}^{n} \frac{2 \lambda_{1, j}^{2}\left(R+R \Lambda_{2}(R) / \Lambda_{1}(R)\right) \theta_{j}\left(r_{j}-\theta_{j}\right)}{\left.\left.R+R \Lambda_{2}(R) / \Lambda_{1}(R)\right)\right)^{1 / n} \lambda_{2, j}(2(R+\mathbf{1})) \lambda_{1, j}^{2}\left(R^{\prime \prime}\right) l_{j}^{2}\left(z^{0}\right)} \\
& =\left(\frac{2 \pi}{3}\right)^{n} \prod_{j=1}^{n} \frac{\theta_{j}\left(r_{j}-\theta_{j}\right)}{\lambda_{2, j}(2(R+\mathbf{1})) l_{j}^{2}\left(z^{0}\right)} .
\end{aligned}
$$

Besides, we have that

$$
\begin{aligned}
& \int_{0}^{\Theta / \mathbf{L}\left(z^{0}\right)} \operatorname{meas}\left\{\mathbb{T}^{n}\left(z^{0}, S\right) \cap G_{R^{\prime}}(F)\right\} d S \\
& \quad=\operatorname{meas}\left\{\mathbb{D}^{n}\left[z^{0}, \frac{\Theta}{\mathbf{L}\left(z^{0}\right)}\right] \cap G_{R^{\prime}}(F)\right\} \\
& \quad \leq \pi^{n} \prod_{j=1}^{n} \frac{\theta_{j}^{2}}{l_{j}^{2}\left(z^{0}\right)} .
\end{aligned}
$$

Hence, the following difference is positive

$$
\begin{aligned}
& \left(\frac{2 \pi}{3}\right)^{n} \prod_{j=1}^{n} \frac{\theta_{j}\left(r_{j}-\theta_{j}\right)}{\lambda_{2, j}(2(R+\mathbf{1})) l_{j}^{2}\left(z^{0}\right)} \\
& -\int_{0}^{\Theta / \mathbf{L}\left(z^{0}\right)} \operatorname{meas}\left\{\mathbb{T}^{n}\left(z^{0}, S\right) \cap G_{R^{\prime}}(F)\right\} d S \\
& \geq\left(\frac{2 \pi}{3}\right)^{n} \prod_{j=1}^{n} \frac{\theta_{j}\left(r_{j}-\theta_{j}\right)}{\lambda_{2, j}(2(R+\mathbf{1})) l_{j}^{2}\left(z^{0}\right)} \\
& -\pi^{n} \prod_{j=1}^{n} \frac{\theta_{j}^{2}}{l_{j}^{2}\left(z^{0}\right)} \\
& =\pi^{n} \prod_{j=1}^{n} \frac{\theta_{j}}{l_{j}^{2}\left(z^{0}\right)} \frac{2 r_{j}-\theta_{j}\left(2+3 \lambda_{2, j}(2(R+\mathbf{1}))\right)}{3 \lambda_{2, j}(2(R+\mathbf{1}))}>0
\end{aligned}
$$

because $\theta_{j}<2 r_{j} /\left(2+3 \lambda_{2, j}(2(R+1))\right)$. From (36) it follows that

$$
\begin{gathered}
\int_{\Theta / \mathbf{L}\left(z^{0}\right)}^{R / \mathbf{L}\left(z^{0}\right)} \operatorname{meas}\left\{\mathbb{T}^{n}\left(z^{0}, S\right) \cap G_{R^{\prime}}(F)\right\} d S \\
<\left(\frac{2 \pi}{3}\right)^{n} \prod_{j=1}^{n} \frac{\theta_{j}\left(r_{j}-\theta_{j}\right)}{\lambda_{2, j}(2(R+\mathbf{1})) l_{j}^{2}\left(z^{0}\right)}
\end{gathered}
$$

$$
\begin{aligned}
& -\int_{0}^{\Theta / \mathbf{L}\left(z^{0}\right)} \operatorname{meas}\left\{\mathbb{T}^{n}\left(z^{0}, S\right) \cap G_{R^{\prime}}(F)\right\} d S \\
& \leq\left(\frac{2 \pi}{3}\right)^{n} \prod_{j=1}^{n} \frac{\theta_{j}\left(r_{j}-\theta_{j}\right)}{\lambda_{2, j}(2(R+\mathbf{1})) l_{j}^{2}\left(z^{0}\right)} .
\end{aligned}
$$

By mean value theorem there exists $R^{0}=R^{0}\left(z^{0}\right)$ with $r_{j} \in$ $\left[\theta_{j}, r_{j}\right]$ such that

$$
\begin{aligned}
& \int_{\Theta / \mathbf{L}\left(z^{0}\right)}^{R / \mathbf{L}\left(z^{0}\right)} \operatorname{meas}\left\{\mathbb{T}^{n}\left(z^{0}, S\right) \cap G_{R^{\prime}}(F)\right\} d S \\
& \quad=\operatorname{meas}\left\{\mathbb{T}^{n}\left(z^{0}, \frac{R^{0}}{\mathbf{L}\left(z^{0}\right)}\right) \cap G_{R^{\prime}}(F)\right\} \prod_{j=1}^{n} \frac{r_{j}-\theta_{j}}{l_{j}\left(z^{0}\right)} .
\end{aligned}
$$

Hence, in view of (39) we obtain a desired inequality

$$
\begin{aligned}
& \text { meas }\left\{\mathbb{T}^{n}\left(z^{0}, \frac{R^{0}}{\mathbf{L}\left(z^{0}\right)}\right) \cap G_{R^{\prime}}(F)\right\} \\
& <\left(\frac{2 \pi}{3}\right)^{n} \prod_{j=1}^{n} \frac{\theta_{j}}{\lambda_{2, j}(2(R+\mathbf{1})) l_{j}\left(z^{0}\right)} .
\end{aligned}
$$

Clearly, for every point $z^{0} \in \mathbb{C}^{n}$ we have $\mathbb{T}^{n}\left(z^{0}, R^{0} / \mathbf{L}\left(z^{0}\right)\right) \backslash$ $Z_{F}=\bigcup_{i} C_{i}^{\prime}$, where $C_{i}^{\prime}$ are connected disjoint sets, $C_{i}^{\prime} \supset C_{i}$ and $C_{i}$ is defined in condition (2). Let $z^{*} \in \mathbb{T}^{n}\left(z^{0}, R / \mathbf{L}\left(z^{0}\right)\right)$ be such that $\mid F\left(z^{*}\right)=\max \left\{|F(z)|: z \in \mathbb{T}^{n}\left(z^{0}, R^{0} / \mathbf{L}\left(z^{0}\right)\right)\right\}$. Then there exists $i_{0}$ such that $z^{*} \in C_{i_{0}}^{\prime}$. Let $z^{* *} \in C_{i_{0}} \subset C_{i_{0}}^{\prime}$ be such that $\left|F\left(z^{* *}\right)\right|=\min _{z \in C_{i_{0}}}|F(z)|$. We choose $J=\left(j_{1}, \ldots, j_{n}\right) \in$ $\mathcal{F}$, where

$$
j_{i}=\left\{\begin{array}{ll}
1, & z_{i}^{*} \neq z_{i}^{* *}, \\
0, & z_{i}^{*}=z_{i}^{* *},
\end{array} \quad i \in\{1, \ldots, n\}\right.
$$


and deduce

$$
\begin{aligned}
\ln \left|\frac{F\left(z^{*}\right)}{F\left(z^{* *}\right)}\right| & \leq \int_{z^{* *}}^{z^{*}}\left|(\ln F(z))^{(J)}\right|\left|d z^{J}\right| \\
& \leq \int_{z^{* *}}^{z^{*}} p_{1} \mathbf{L}^{J}(z)\left|d z^{J}\right| \\
& \leq p_{1} \mathbf{L}^{J}\left(z^{0}\right) \Lambda_{2}^{J}(R) \cdot \frac{(\pi R)^{J}}{\mathbf{L}^{J}\left(z^{0}\right)} \\
& \leq \pi^{n} p_{1} R^{J} \Lambda_{2}^{J}(R) .
\end{aligned}
$$

Hence,

$$
\begin{aligned}
\max & \left\{|F(z)|: z \in \mathbb{T}^{n}\left(z^{0}, \frac{R^{0}}{\mathbf{L}\left(z^{0}\right)}\right)\right\}=\left|F\left(z^{*}\right)\right| \\
& \leq \exp \left\{\pi^{n} p_{1} R^{J} \Lambda_{2}^{J}(R)\right\}\left|F\left(z^{* *}\right)\right| \\
& =\exp \left\{\pi^{n} p_{1} R^{J} \Lambda_{2}^{J}(R)\right\} \min _{z \in C_{i_{0}}}|F(z)| \\
& \leq \exp \left\{\pi^{n} p_{1} R^{J} \Lambda_{2}^{J}(R)\right\} p_{2} \min _{i} \min _{z \in C_{i}}|F(z)| \\
& =\exp \left\{\pi^{n} p_{1} R^{J} \Lambda_{2}^{J}(R)\right\} p_{2} \\
& \cdot \min \left\{|F(z)|: z \in \mathbb{T}^{n}\left(z^{0}, \frac{R^{0}}{\mathbf{L}\left(z^{0}\right)}\right) \backslash G_{R^{\prime}}(F)\right\} .
\end{aligned}
$$

By Theorem 7 the function $F$ has bounded L-index in joint variables.

Let us to denote $\Delta$ as Laplace operator. We will consider $\Delta \ln |F|$ as generalized function. Using some known results from potential theory, we can rewrite Theorem 9 as follows.

Theorem 10. Let $\mathbf{L} \in Q^{n}$. If an entire function $F$ satisfies the following conditions

(1) for every $R>\mathbf{0}$ there exists $p_{1}=p_{1}(R)>0$ such that for all $z \in \mathbb{C}^{n} \backslash G_{R}(F)$ and for every $j \in\{1, \ldots, n\}$

$$
\left|\frac{\partial \ln F(z)}{\partial z_{j}}\right| \leq p_{1} l_{j}(z)
$$

where $\ln F(z)$ is the principal value of logarithm.

(2) for every $R>\mathbf{0}$ and $R^{\prime} \geq \mathbf{0}$ exists $p_{2}=$ $p_{2}\left(R, R^{\prime}\right) \geq 1$ that for all $z^{0} \in \mathbb{C}^{n}$ such that $T^{n}\left(z^{0}, R / \mathbb{L}\left(z^{0}\right)\right) \backslash G_{R^{\prime}}(F)=\bigcup_{i} C_{i} \neq \emptyset$, where the sets $C_{i}$ are connected disjoint sets, and either (a) $\max _{i} \min _{z \in C_{i}}|F(z)| \leq p_{2} \min _{i} \min _{z \in C_{i}}|F(z)|$, or (b) $\max _{i} \max _{z \in C_{i}}|F(z)| \leq p_{2} \min _{i} \max _{z \in C_{i}}|F(z)|$, or (c) $\left|F\left(z^{*}\right)\right|=\max _{i} \max _{z \in C_{i}}|F(z)|,\left|F\left(z^{* *}\right)\right|=$ $\min _{i} \min _{z \in C_{i}}|F(z)|$, and $z^{*}, z^{* *}$ belong to the same set $C_{i_{0}}$

(3) for every $R>0$ there exists $n^{*}(R)>0$ such that for all $z \in \mathbb{C}^{n}$

$$
\int_{\mathbb{D}^{n}\left[z^{0}, R / \mathbf{L}\left(z^{0}\right)\right]} \Delta \ln |F| d V_{2 n} \leq n^{*}(R)
$$

then $F$ has bounded $\mathbf{L}$-index in joint variables.
Proof. Ronkin [28, p. 230] deduced the following formula for entire function:

$$
\int_{\mathbb{D}^{n}\left[0, R^{*}\right]} \Delta \ln |F| d V_{2 n}=2 \pi \int_{Z_{F} \cap \mathbb{D}^{n}\left[0, R^{*}\right]} \gamma_{F}(z) d V_{2 n-2},
$$

where $\gamma_{F}(z)$ is a multiplicity of zero point of the function $F$ at point $z, R^{*} \in \mathbb{R}_{+}^{n}$ is arbitrary radius. Let $\chi_{F}(z)$ be a characteristic function of zero set of $F$. Then $\chi_{F}(z) \leq \gamma_{F}(z)$. Hence,

$$
\begin{aligned}
& \text { meas }\left\{Z_{F} \cap \mathbb{D}^{n}\left[z^{0}, \frac{R}{\mathbf{L}\left(z^{0}\right)}\right]\right\} \\
& \quad=\int_{Z_{F} \cap \mathbb{D}^{n}\left[z^{0}, R / \mathbf{L}\left(z^{0}\right)\right]} \chi_{F}(z) d V_{2 n-2} \\
& \quad \leq \int_{Z_{F} \cap \mathbb{D}^{n}\left[z^{0}, R / \mathbf{L}\left(z^{0}\right)\right]} \gamma_{F}(z) d V_{2 n-2} \\
& \quad=\frac{1}{2 \pi} \int_{\mathbb{D}^{n}\left[z^{0}, R / \mathbf{L}\left(z^{0}\right)\right]} \Delta \ln |F| d V_{2 n} \leq \frac{n^{*}(R)}{2 \pi} ;
\end{aligned}
$$

that is, inequality (29) holds.

Now we want to prove that (45) implies (28). For every $J \in \mathscr{J} \backslash \bigcup_{k=1}^{n} \mathbf{1}_{k}$ and $z^{0} \in \mathbb{C}^{n} \backslash G_{R}(F)$, Cauchy's integral formula can be written in the following form

$$
\begin{aligned}
& \left(\ln F\left(z^{0}\right)\right)^{(J)} \\
& \quad=\frac{J !}{(2 \pi i)^{n}} \int_{\mathbb{T}^{n}\left(z^{0}, R / \mathbf{L}\left(z^{0}\right)\right)} \frac{(\ln F(z))^{\left(\mathbf{1}_{m}\right)}}{\left(z-z^{0}\right)^{J-\mathbf{1}_{m}+\mathbf{1}}} d z,
\end{aligned}
$$

where $m$ is such that $j_{m}=1$.

If $z^{0} \in \mathbb{C}^{n} \backslash G_{R}(F)$ and $z^{\prime} \in Z_{F} \subset G_{R}(F)$, then for every $j \in\{1, \ldots, n\}$

$$
\left|z_{j}^{0}-z_{j}^{\prime}\right| \geq \frac{r_{j}}{l_{j}\left(z^{\prime}\right)} \geq \frac{r_{j} \lambda_{1, j}(R)}{l_{j}\left(z^{0}\right)}>\frac{r_{j} \lambda_{1, j}(R)}{2 l_{j}\left(z^{0}\right)} .
$$

Let us consider the set $A=\bigcup_{z^{0} \in \mathbb{C}^{n} \backslash G_{R}(F)} \mathbb{T}^{n}\left(z^{0}, R \Lambda_{1}(R) /\right.$ $2 \mathbf{L}\left(z^{0}\right)$ ). We want to find the greatest radius $R^{*} \in \mathbb{R}_{+}^{n}$ such that $G_{R^{*}}(F) \cap A=\emptyset$ :

$$
\begin{aligned}
\frac{R}{\mathbf{L}\left(z^{\prime}\right)}-\frac{R \Lambda_{1}(R)}{2 \mathbf{L}\left(z^{0}\right)} & \geq \frac{R}{\mathbf{L}\left(z^{\prime}\right)}-\frac{R \Lambda_{1}(R)}{2 \Lambda_{1}(R) \mathbf{L}\left(z^{\prime}\right)} \\
& =\frac{R}{2 \mathbf{L}\left(z^{\prime}\right)}
\end{aligned}
$$


Thus, for $R^{*}=R / 3 A \subset \mathbb{C}^{n} \backslash G_{R^{*}}(F)$. Using (45), we obtain that for every $z^{0} \in \mathbb{C}^{n} \backslash G_{R}(F)$

$$
\begin{aligned}
& \left|\left(\ln F\left(z^{0}\right)\right)^{(J)}\right| \leq \frac{1}{(2 \pi)^{n}} \\
& \cdot \int_{\mathbb{J}^{n}\left(z^{0}, R \Lambda_{1}(R) / 2 \mathbf{L}\left(z^{0}\right)\right)} \frac{\left|(\ln F(z))^{\left(\mathbf{1}_{m}\right)}\right|}{\left|z-z^{0}\right|^{J-\mathbf{1}_{m}+\mathbf{1}}}|d z| \leq \frac{1}{(2 \pi)^{n}} \\
& \cdot \int_{\mathbb{V}^{n}\left(z^{0}, R \Lambda_{1}(R) / 2 \mathbf{L}\left(z^{0}\right)\right)}\left(\frac{2 \mathbf{L}\left(z^{0}\right)}{R \Lambda_{1}(R)}\right)^{J-\mathbf{1}_{m}+\mathbf{1}} p_{1}\left(\frac{1}{3} R\right) \\
& \cdot l_{m}(z)|d z| \leq \frac{1}{(2 \pi)^{n}} \\
& \cdot \int_{\mathbb{T}^{n}\left(z^{0}, R \Lambda_{1}(R) / 2 \mathbf{L}\left(z^{0}\right)\right)}\left(\frac{2 \mathbf{L}\left(z^{0}\right)}{R \Lambda_{1}(R)}\right)^{J-\mathbf{1}_{m}+\mathbf{1}} p_{1}\left(\frac{1}{3} R\right) \\
& \cdot \lambda_{2, m}\left(0.5 R \Lambda_{1}(R)\right) l_{m}\left(z^{0}\right)|d z| \\
& =\frac{r_{m} \lambda_{1, m}(R)}{2(2 \pi)^{n}}\left(\frac{2 \mathbf{L}\left(z^{0}\right)}{R \Lambda_{1}(R)}\right)^{J+\mathbf{1}} p_{1}\left(\frac{1}{3} R\right) \\
& \cdot \lambda_{2, m}\left(0.5 R \Lambda_{1}(R)\right) \int_{\mathbb{T}^{n}\left(z^{0}, R \Lambda_{1}(R) / 2 \mathbf{L}\left(z^{0}\right)\right)}|d z| \\
& =0.5 r_{m} \lambda_{1, m}(R) p_{1}\left(\frac{1}{3} R\right) \lambda_{2, m}\left(0.5 R \Lambda_{1}(R)\right) \\
& \left(\frac{2 \mathbf{L}\left(z^{0}\right)}{R \Lambda_{1}(R)}\right)^{J} \leq C(R) \mathbf{L}^{J}\left(z^{0}\right),
\end{aligned}
$$

where $C(R)=0.5 p_{1}((1 / 3) R) \max _{J \in \mathcal{F}}\left\{\lambda_{2, m}\left(0.5 R \Lambda_{1}(R)\right) r_{m}\right.$ $\left.\lambda_{1, m}(R)\left(2 / R \Lambda_{1}(R)\right)^{J}\right\}$. Thus, we proved that inequality (28) is valid.

For $n=1$ Theorem 9 implies the following corollary.

Corollary 11. Let $l \in Q, f$ be an entire in $\mathbb{C}$ function, $n\left(r, z^{0}, f\right)$ a number of zeros of the $f$ in the disc $\left|z-z_{0}\right| \leq$ $r / l\left(z^{0}\right)$. If the function $f$ satisfies the following conditions:

(1) for every $r>0$ there exists $p_{1}=p_{1}(r)>0$ such that for all $z \in \mathbb{C} \backslash G_{r}(f)$

$$
\left|\frac{f^{\prime}(z)}{f(z)}\right| \leq p_{1} l(z),
$$

(2) for every $r>0$ and $r^{\prime} \geq 0$ exists $p_{2}=p_{2}\left(r, r^{\prime}\right) \geq$ 1 that for all $z^{0} \in \mathbb{C}$ such that $\{z \in \mathbb{C}$ : $\left.\left|z-z^{0}\right|=r / l\left(z^{0}\right)\right\} \backslash G_{r^{\prime}}(f)=\bigcup_{i} C_{i} \neq \emptyset$, where the sets $C_{i}$ are connected disjoint sets, and either (a) $\max _{i} \min _{z \in C_{i}}|f(z)| \leq p_{2} \min _{i} \min _{z \in C_{i}}|f(z)|$, or (b) $\max _{i} \max _{z \in C_{i}}|f(z)| \leq p_{2} \min _{i} \max _{z \in C_{i}}|f(z)|$, or (c) $\left|f\left(z^{*}\right)\right|=\max _{i} \max _{z \in C_{i}}|f(z)|,\left|f\left(z^{* *}\right)\right|=$ $\min _{i} \min _{z \in C_{i}}|f(z)|$, and $z^{*}, z^{* *}$ belong to the same set $C_{i_{0}}$
(3) for every $r>0$ there exists $n^{*}(r)>0$ such that for all $z^{0} \in \mathbb{C} n\left(r, z^{0}, f\right) \leq n^{*}(r)$,

then $f$ has bounded l-index.

It is known (see $[12,27,29])$ that in one-dimensional case conditions (1) and (3) of Corollary 11 are necessary and sufficient for boundedness of $l$-index or index. Thus, condition (2) is excessive in the case. But for $\mathbb{C}^{n}(n \geq 2)$, it is required because $\mathbb{D}^{n}\left[z^{0}, R / \mathbf{L}\left(z^{0}\right)\right] \backslash G_{R^{\prime}}(F)$ is a multiply connected domain, when $\mathbb{D}^{n}\left[z^{0}, R / \mathbf{L}\left(z^{0}\right)\right]$ contains zeros of the function $F$.

We need some notations from [1]. Let $\mathbf{b} \in \mathbb{C}^{n} \backslash\{\mathbf{0}\}$ be a given direction. For a given $z^{0} \in \mathbb{C}^{n}$ we denote $g_{z^{0}}(t):=$ $F\left(z^{0}+t \mathbf{b}\right)$. If one has $g_{z^{0}}(t) \neq 0$ for all $t \in \mathbb{C}$, then $G_{r}^{\mathbf{b}}\left(F, z^{0}\right):=$ $\emptyset$; if $g_{z^{0}}(t) \equiv 0$, then $G_{r}^{\mathbf{b}}\left(F, z^{0}\right):=\left\{z^{0}+t \mathbf{b}: t \in \mathbb{C}\right\}$. And if $g_{z^{0}}(t) \not \equiv 0$ and $a_{k}^{0}$ are zeros of the function $g_{z^{0}}(t)$, then $G_{r}^{\mathbf{b}}\left(F, z^{0}\right):=\bigcup_{k}\left\{z^{0}+t \mathbf{b}:\left|t-a_{k}^{0}\right| \leq r / L\left(z^{0}+a_{k}^{0} \mathbf{b}\right)\right\}, r>0$. Let

$$
G_{r}^{\mathbf{b}}(F)=\bigcup_{z^{0} \in \mathbb{C}^{n}} G_{r}^{\mathbf{b}}\left(F, z^{0}\right)
$$

Remark 12. In [1, Theorem 8], sufficient conditions of boundedness of $\mathbf{L}$-index in joint variables were obtained, which are similar to Theorem 10. Particularly, we assumed the validity of inequality (45) for all $z \in \mathbb{C}^{n} \backslash G_{r_{j}}^{\mathbf{1}_{j}}(F), j \in\{1,2, \ldots, n\}$. However, $G_{r_{j}}^{\mathbf{1}_{j}}(F) \subset G_{R}(F)$, where $R=\left(r_{1}, \ldots, r_{n}\right)$. Thus, condition (1) in Theorem 10 is weaker than the corresponding assumption in Theorem 8 from [1].

\section{Conflicts of Interest}

The authors declare that they have no conflicts of interest.

\section{References}

[1] A. I. Bandura, M. T. Bordulyak, and O. B. Skaskiv, "Sufficient conditions of boundedness of L-index in joint variables," Matematychni Studii, vol. 45, no. 1, pp. 12-26, 2016.

[2] A. I. Bandura and O. B. Skaskiv, "Open problems for entire functions of bounded index in direction," Matematychni Studii, vol. 43, no. 1, pp. 103-109, 2015.

[3] A. Bandura and O. Skaskiv, Entire Functions of Several Variables of Bounded Index, I. E. Chyzhykov, Ed., Chyslo, Publisher, Lviv, Ukraine, 2016, http://chyslo.com.ua/.

[4] B. C. Chakraborty and T. K. Samanta, "On entire functions of bounded index in several complex variables," Journal of Pure Mathematics, vol. 17, pp. 53-71, 2000.

[5] G. J. Krishna and S. M. Shah, "Functions of bounded indices in one and several complex variables," in Mathematical Essays Dedicated to A. J. Macintyre, pp. 223-235, Ohio Univ. Press, Athens, Ohio, USA, 1970.

[6] F. Nuray and R. F. Patterson, "Entire bivariate functions of exponential type," Bulletin of Mathematical Sciences, vol. 5, no. 2, pp. 171-177, 2015.

[7] F. Nuray and R. F. Patterson, "Multivalence of bivariate functions of bounded index," Le Matematiche, vol. 70, no. 2, pp. 225233, 2015. 
[8] R. F. Patterson and F. Nuray, "A characterization of holomorphic bivariate functions of bounded index," Mathematica Slovaca, vol. 67, no. 3, pp. 731-736, 2017.

[9] M. T. Bordulyak, "A proof of Sheremeta conjecture concerning entire function of bounded l-index," Matematychni Studii, vol. 12, no. 1, pp. 108-110, 1999.

[10] A. Bandura, O. Skaskiv, and P. Filevych, "Properties of entire solutions of some linear PDE's," Journal of Applied Mathematics and Computational Mechanics, vol. 16, no. 2, pp. 17-28, 2017.

[11] A. Bandura and O. Skaskiv, "Entire functions of several variables of bounded L-index in direction and of bounded index in joint variables," 2015, https://arxiv.org/abs/1508.07486.

[12] M. Sheremeta, Analytic Functions of Bounded Index, VNTL Publishers, Lviv, Ukraine, 1999.

[13] I. Chyzhykov, G. G. Gundersen, and J. Heittokangas, "Linear differential equations and logarithmic derivative estimates," Proceedings of the London Mathematical Society. Third Series, vol. 86, no. 3, pp. 735-754, 2003.

[14] I. Chyzhykov, J. Heittokangas, and J. Rättyä, "Sharp logarithmic derivative estimates with applications to ordinary differential equations in the unit disc," Journal of the Australian Mathematical Society, vol. 88, no. 2, pp. 145-167, 2010.

[15] J. Heittokangas, R. Korhonen, and J. Rättyä, "Generalized logarithmic derivative estimates of gol'dberg and grinshtein type," Bulletin of the London Mathematical Society, vol. 36, no. 1, pp. 105-114, 2004.

[16] J. Heittokangas, "Growth estimates for logarithmic derivatives of Blaschke products and of functions in the Nevanlinna class," Kodai Mathematical Journal, vol. 30, no. 2, pp. 263-279, 2007.

[17] J. Agler, J. E. McCarthy, and M. Stankus, "Local geometry of zero sets of holomorphic functions near the torus," New York Journal of Mathematics, vol. 14, pp. 517-538, 2008.

[18] H. Ahn and H. R. Cho, "Zero sets of holomorphic functions in the Nevanlinna type class on convex domains in C2," Japanese Journal of Mathematics, vol. 28, no. 2, pp. 245-260, 2002.

[19] A. Cumenge, "Zero sets of functions in the Nevanlinna or the Nevanlinna-Djrbachian classes," Pacific Journal of Mathematics, vol. 199, no. 1, pp. 79-92, 2001.

[20] M. Jasiczak, "Remark on zero sets of holomorphic functions in convex domains of finite type," Canadian Mathematical Bulletin. Bulletin Canadien de Mathématiques, vol. 53, no. 2, pp. 311-320, 2010.

[21] M. Kanter, "Gauss-Lucas theorems for entire functions on $C^{M}$," Complex Variables and Elliptic Equations. An International Journal, vol. 60, no. 1, pp. 93-98, 2015.

[22] J. Lundqvist, "An explicit calculation of the Ronkin function," Annales de la Faculté des Sciences de Toulouse, vol. 24, no. 2, pp. 227-250, 2015.

[23] A. Bandura, M. Bordulyak, and O. Skaskiv, "Sufficient conditions of boundedness of L-index in joint variables," Matematychni Studii, vol. 45, no. 1, pp. 12-26, 2016.

[24] M. T. Bordulyak and M. M. Sheremeta, "Boundedness of the L-index of an entire function of several variables," Dopividi Akademiï Nauk Ukraïni, no. 9, pp. 10-13, 1993.

[25] M. Salmassi, "Functions of bounded indices in several variables," Indian Journal of Mathematics, vol. 31, no. 3, pp. 249-257, 1989.

[26] G. H. Fricke, "Entire functions of locally slow growth," Journal d'Analyse Mathematique, vol. 28, no. 1, pp. 101-122, 1975.

[27] M. N. Sheremeta and A. D. Kuzyk, "Logarithmic derivative and zeros of an entire function of bounded l-index," Siberian Mathematical Journal, vol. 33, no. 2, pp. 304-312, 1992.
[28] L. I. Ronkin, "Introduction to theory of entire functions of several variables," Translations of Mathematical Monographs, vol. 44, 273 pages, 1974.

[29] G. H. Fricke, "Functions of bounded index and their logarithmic derivatives," Mathematische Annalen, vol. 206, pp. 215-223, 1973. 


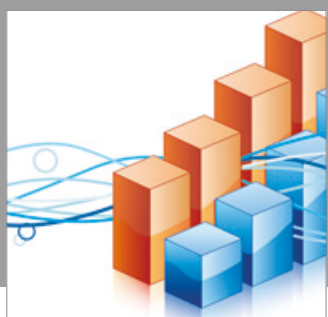

Advances in

Operations Research

vatersals

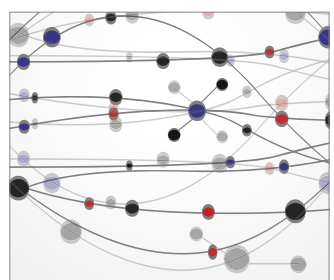

\section{The Scientific} World Journal
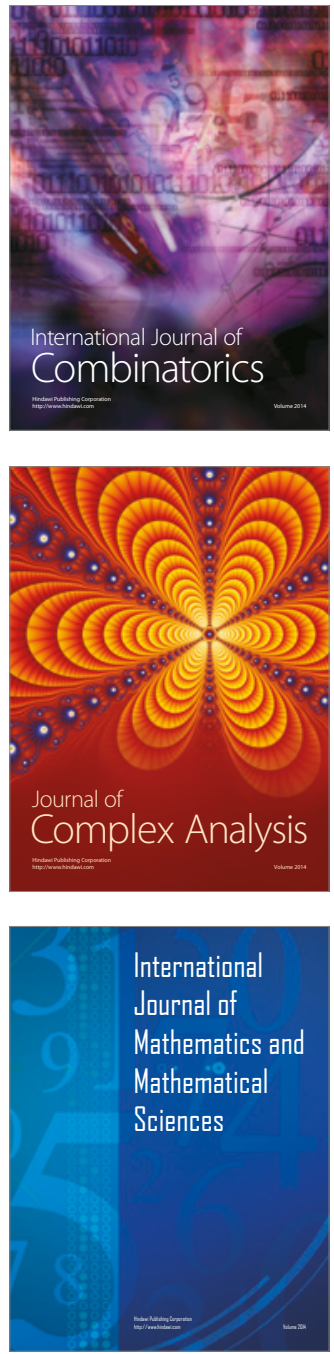
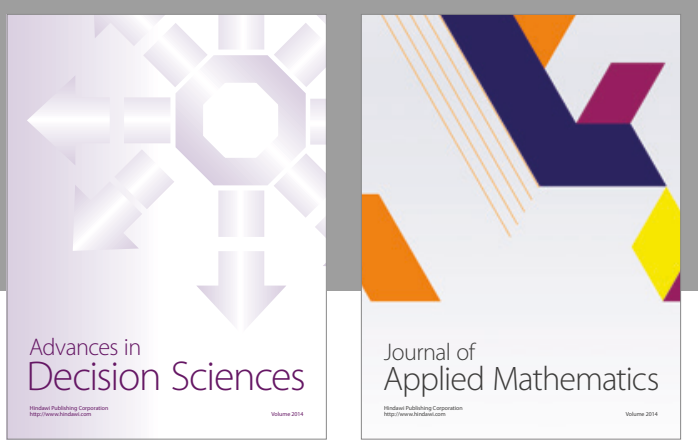

Algebra

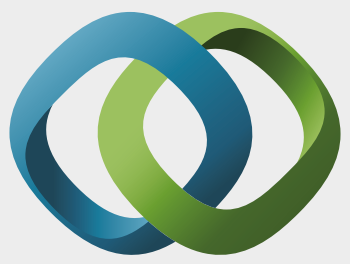

\section{Hindawi}

Submit your manuscripts at

https://www.hindawi.com
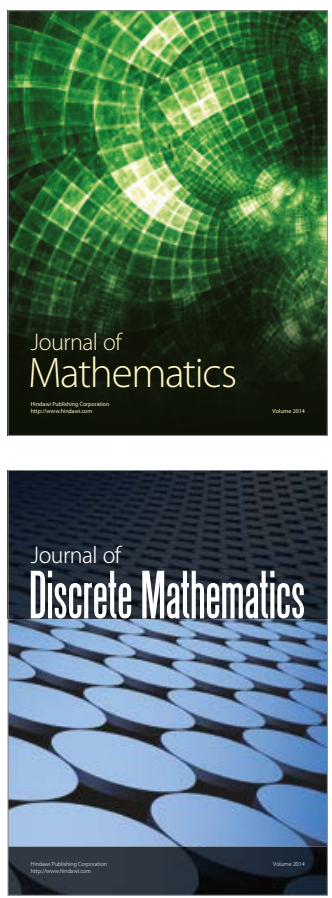

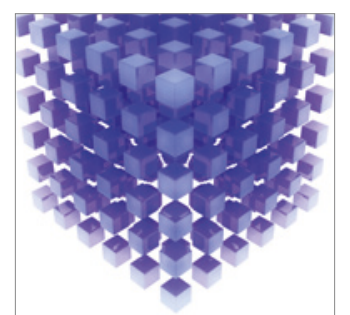

Mathematical Problems in Engineering
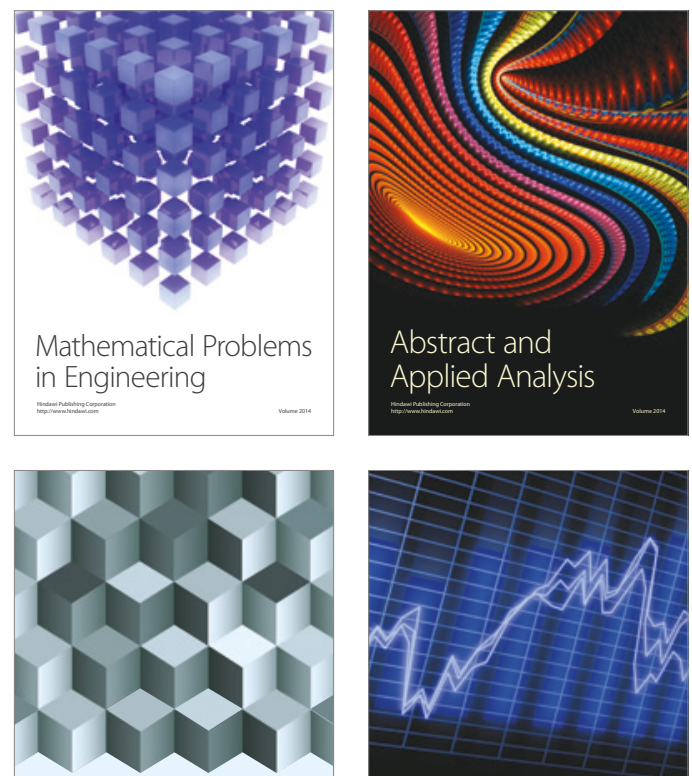

Journal of

Function Spaces

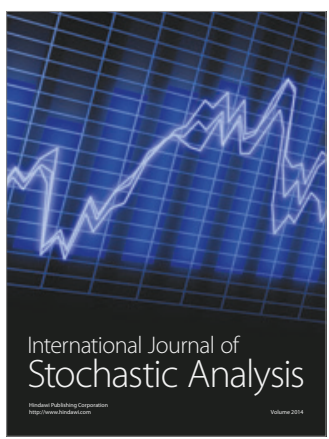

Probability and Statistics
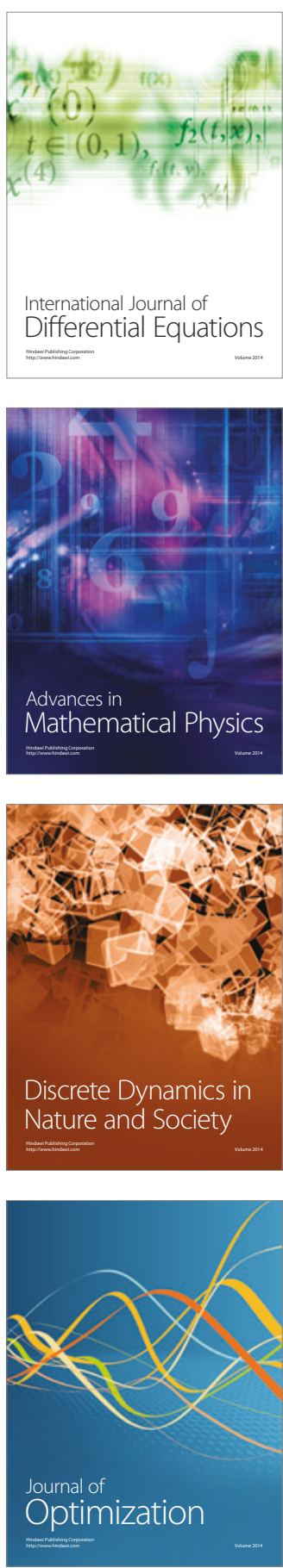